\title{
Oligonucleotide microarray analysis reveals dysregulation of energy-related metabolism in insulin-sensitive tissues of type 2 diabetes patients
}

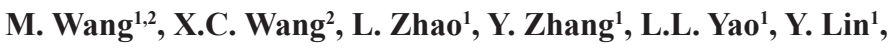 \\ Y.D. Peng ${ }^{1}$ and R.M. $\mathrm{Hu}^{2}$ \\ ${ }^{1}$ Department of Endocrinology and Metabolism, Shanghai Jiaotong University, \\ Shanghai Peoples Hospital Affiliated, Shanghai, China \\ ${ }^{2}$ Department of Endocrinology, Huashan Hospital, \\ Institute of Endocrinology and Diabetology at Fudan University, \\ Shanghai Medical College, Fudan University, Shanghai, China \\ Corresponding authors: Y.D. Peng / R.M. Hu \\ E-mail: pyongde@hotmail.com / renminghu@fudan.edu.cn
}

Genet. Mol. Res. 13 (2): 4494-4504 (2014)

Received January 14, 2013

Accepted June 10, 2013

Published June 17, 2014

DOI http://dx.doi.org/10.4238/2014.June.17.1

\begin{abstract}
Impaired insulin action within skeletal muscle, adipose tissue, and the liver is an important characteristic of type 2 diabetes (T2D). In order to identify common underlying defects in insulinsensitive tissues that may be involved in the pathogenesis of T2D, the gene expression profiles of skeletal muscle, visceral adipose tissue, and liver from autopsy donors with or without T2D were examined using oligonucleotide microarrays and quantitative reverse transcriptasePCR. Compared with controls, 691 genes were commonly dysregulated in these three insulin-sensitive tissues of humans with T2D. These co-expressed genes were enriched within the mitochondrion, with suggested involvement in energy metabolic processes such as glycolysis and gluconeogenesis, fatty acid beta oxidative, tricarboxylic acid cycle,
\end{abstract}


and electron transport. Genes related to energy metabolism were mostly downregulated in diabetic skeletal muscle and visceral adipose tissue, while they were upregulated in the diabetic liver. This observed dysregulation in energy-related metabolism may be the underlying factor leading to the molecular mechanisms responsible for the insulin resistance of patients with $\mathrm{T} 2 \mathrm{D}$.

Key words: Oligonucleotide microarray; Insulin-sensitive tissues; Type 2 diabetes; Energy metabolism

\section{INTRODUCTION}

Insulin resistance within insulin-sensitive tissues, including skeletal muscle, adipose tissue, and liver, is a cardinal characteristic of individuals with fully developed type 2 diabetes (T2D) (Kasuga, 2006). Not all insulin-resistant subjects, however, develop T2D. Since the development of T2D is likely also partly determined by genetic factors, understanding the gene expression profiles of insulin-sensitive tissues from diabetic animals and humans may help to uncover the underlying molecular mechanisms involved in the disease.

Studies using microarrays have been performed in an effort to identify candidate genes or pathways involved in insulin resistance and diabetes, in both animal and human models. In addition to microarray studies on a single type of insulin-sensitive tissue (Lowell and Shulman, 2005; Wisloff et al., 2005; Dahlman et al., 2006), gene expression profiles of three insulin-sensitive tissues, skeletal muscle, adipose tissue, and liver, have been recently reported. The activation of the peroxisome proliferator-activated receptor $\gamma(\operatorname{PPAR} \gamma)$, a factor implicated in energy metabolism, has effects on gene expression in three multiple insulinsensitive tissues (Way et al., 2001). Genes encoding $\beta$-oxidation and electron transport chain components have also been shown to be simultaneously upregulated in adipose tissue, heart, liver, and skeletal muscle of perilipin knockout mice (Castro-Chavez et al., 2003). Suh et al. (2005) analyzed the gene expression profiles within insulin-sensitive tissues from pre-diabetic and diabetic Zucker diabetic fatty rats (Suh et al., 2005). Energy restriction was found to affect gene expression in skeletal muscle, liver, fat, and pancreatic islets of male Zucker diabetic fatty rats (Colombo et al., 2005, 2006). In lean and obese diabetes-resistant and diabetessusceptible mice, the co-expression of genes within insulin target tissues was linked with cell cycle regulation (Keller et al., 2008). Recently, an unconventional microarray design revealed that the response to obesity is largely tissue specific in insulin-sensitive tissues that react to dietary-induced diabetes (Lee et al., 2012). Due to the difficulty of simultaneously obtaining these different samples from humans with diabetes, however, there is currently no comprehensive report of gene expression within liver, skeletal muscle, and visceral adipose tissue of a T2D individual.

In the present study, using oligonucleotide microarrays, we characterized gene expression profiles of insulin-sensitive tissues from patients with T2D. We demonstrated that genes with altered expression in all three insulin-sensitive tissues were predominately grouped in the mitochondrial categories of cellular components of gene ontology (GO), and were involved in electron transport, the tricarboxylic acid (TCA) cycle, fatty acid metabolism, and glycolysis and gluconeogenesis. Simultaneously, we identified a proportion of the genes involved in oxi- 
dative metabolism with quantitative reverse transcriptase (qRT)-PCR. This method, therefore, identified several candidate genes and molecular mechanisms that may be responsible for the insulin resistance and progression of $\mathrm{T} 2 \mathrm{D}$.

\section{MATERIAL AND METHODS}

\section{Human tissues}

Human specimens of liver, visceral adipose tissue, and skeletal muscle were obtained from the Tissue Bank at Shanghai Medical College, Fudan University. The donors of these organs had given written consent while alive, and their families had signed off on their consent after their death. The study was approved by the Ethics Committee of the Huashan Hospital, Fudan University, China. Post-mortem processing of tissue was performed according to the Tissue Bank protocol. These three tissues were isolated from donors within $3 \mathrm{~h}$ after death, flash-frozen in liquid nitrogen, and stored at $-80^{\circ} \mathrm{C}$. Tissues were divided for two sets of data. DNA chip analysis data were obtained from one patient with T2D and one without T2D, men that were 55 and 50 years old, respectively. qRT-PCR analysis data were obtained from one woman and three men patients with T2D (age $=72$ years \pm 3.72$)$ and one woman and four men without T2D (age $=52 \pm 11.1)(\mathrm{P}=0.207)$.

\section{RNA isolation and microarray analysis}

Total RNA was extracted from livers, visceral adipose tissues, and gastrocnemius muscle using Trizol (Invitrogen, Carlsbad, CA, USA), purified with RNeasy columns (Qiagen, Valencia, CA, USA), and synthesized to biotin-labeled cRNA according to the standard Affymetrix protocol. The resultant product was hybridized to Human Genome HG U133 Plus 2.0 microarrays (Affymetrix, Santa Clara, CA, USA) containing 54,675 probe-sets representing around 22,000 unique genes. Six separate arrays were run for the samples tested, and each array was replicated twice. Arrays were scanned using a Hewlett Packard Gene Array scanner. Intensity values were quantified using the MAS 5.0 software (Affymetrix).

\section{Normalization, differential expression analysis, and functional annotation analysis}

Raw array data were normalized using toolbox in MATLAB to remove background bias. The differential expression of genes between diabetic and control groups was evaluated by fold-change. Genes that were differently expressed in skeletal muscle, adipose tissue, and liver were tested for over-representation of functional annotation categories, including GO and Kyoto Encyclopedia of Genes and Genomes (KEGG) pathways, using tools within the Database for Annotation, Visualization and Integrated Discovery (DAVID) (http://niaid.abcc. ncifcrf.gov/) and gene set-enrichment analysis (GSEA) (Dennis Jr. et al., 2003; Subramanian et al., 2005; Huang et al., 2009).

\section{qRT-PCR}

The observed differential expression of selected genes identified by microarray analy- 
sis was validated by SYBR Green qRT-PCR. Total RNA $(1 \mu \mathrm{g})$ from study samples was reverse transcribed with reverse transcriptase ReverTra Ace (TOYOBO, Japan). For each qRT-PCR, 1 $\mu \mathrm{L}$ was amplified in a $15-\mu \mathrm{L}$ reaction using a SYBR ${ }^{\circledR}$ Premix Ex Taq ${ }^{\mathrm{TM}}$ Perfect Real-Time Kit (TaKaRa, Japan) according to manufacturer instructions. Samples were run in the ABI Prism 7900HT Sequence Detection System (Applied Biosystems, Foster City, CA, USA) with an initial denaturation step at $95^{\circ} \mathrm{C}$ for $10 \mathrm{~min}$, followed by 40 cycles consisting of denaturation at $95^{\circ} \mathrm{C}$ for $5 \mathrm{~s}$ and annealing at $60^{\circ} \mathrm{C}$ for $30 \mathrm{~s}$. $\beta$-actin expression was used to normalize target gene amplification values. Melting curve profiles and agarose gel electrophoresis analysis were performed to confirm the specificity of the amplification. The relative amounts of PCR product were determined using the comparative $\mathrm{Ct}$ method $\left(2^{-\Delta \Delta \mathrm{Ct}}\right)$.

\section{RESULTS}

\section{Global gene expression profile of insulin-sensitive tissues from a human with T2D}

Of about 54,000 probes represented on the array, 9055 probes were selected following the removal of low-quality probes $(\mathrm{P}>0.05)$ and normalization with the 'manorm' function in MATLAB. With a cutoff value for differential expression of 1.5 -fold, the numbers of differentially expressed probes in diabetic liver, adipose tissue, and skeletal muscle were as shown in Figure 1. Seven hundred and thirty-five probes (691 known genes) were differentially expressed in all three insulin-sensitive tissues, including 66 (58 known genes) that were coupregulated and 204 (187 known genes) that were co-downregulated. Further analysis focused on all the 735 probes identified to be differentially expressed in these insulin-sensitive tissues.

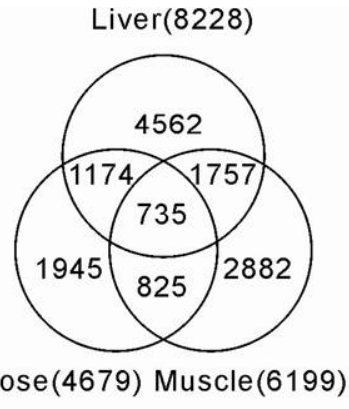

A:all probes

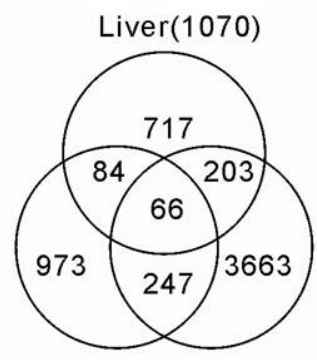

Adipose(1370) Muscle(4179)

B:up-regulated probes

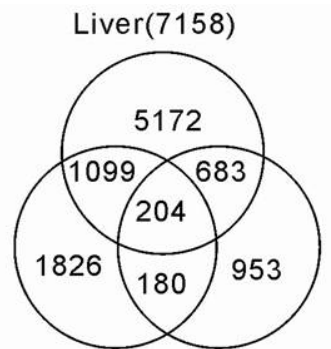

Adipose(3309) Muscle(2020)

C:down-regulated probes

Figure 1. Venn diagrams of probe sets exhibiting differential expression in liver, adipose tissue, and skeletal muscle of human with T2DM. A. An aggregate of all differentially expressed probe sets; B. upregulated probe sets; C. downregulated probe sets. Note that the left panel was not the sum of the another two panels because some probe sets showed divergent effects, being upregulated in one tissue and downregulated in another.

\section{GO categories for genes differentially expressed in various insulin-sensitive tissues from a human with T2D}

To assess the function of the 735 probes differentially expressed in all the insulinsensitive tissues, we examined the Cell Component, Biological Process, and Molecular Func- 
tion GO categories that were used with the GO Chart feature offered by DAVID, as shown in Figure 2. Many co-enzyme binding $\left(\mathrm{P}=2.75 \times 10^{-4}\right)$, electron carrier $(\mathrm{P}=0.021)$, and oxidoreductase activities $(\mathrm{P}=0.025)$ were enriched for with those $\mathrm{GO}$ molecular function terms $(\mathrm{P}$ $<0.05$; Figure 2A). Moreover, the mitochondrial categories within the cellular component of GO were also often found (Figure 2B). Similarly, several energy metabolism-related GO biological process terms were significantly overrepresented $(\mathrm{P}<0.001$; Figure $2 \mathrm{C})$. These findings indicated that $\mathrm{GO}$ categories for these differential expressed probes were predominantly related to energy metabolism.

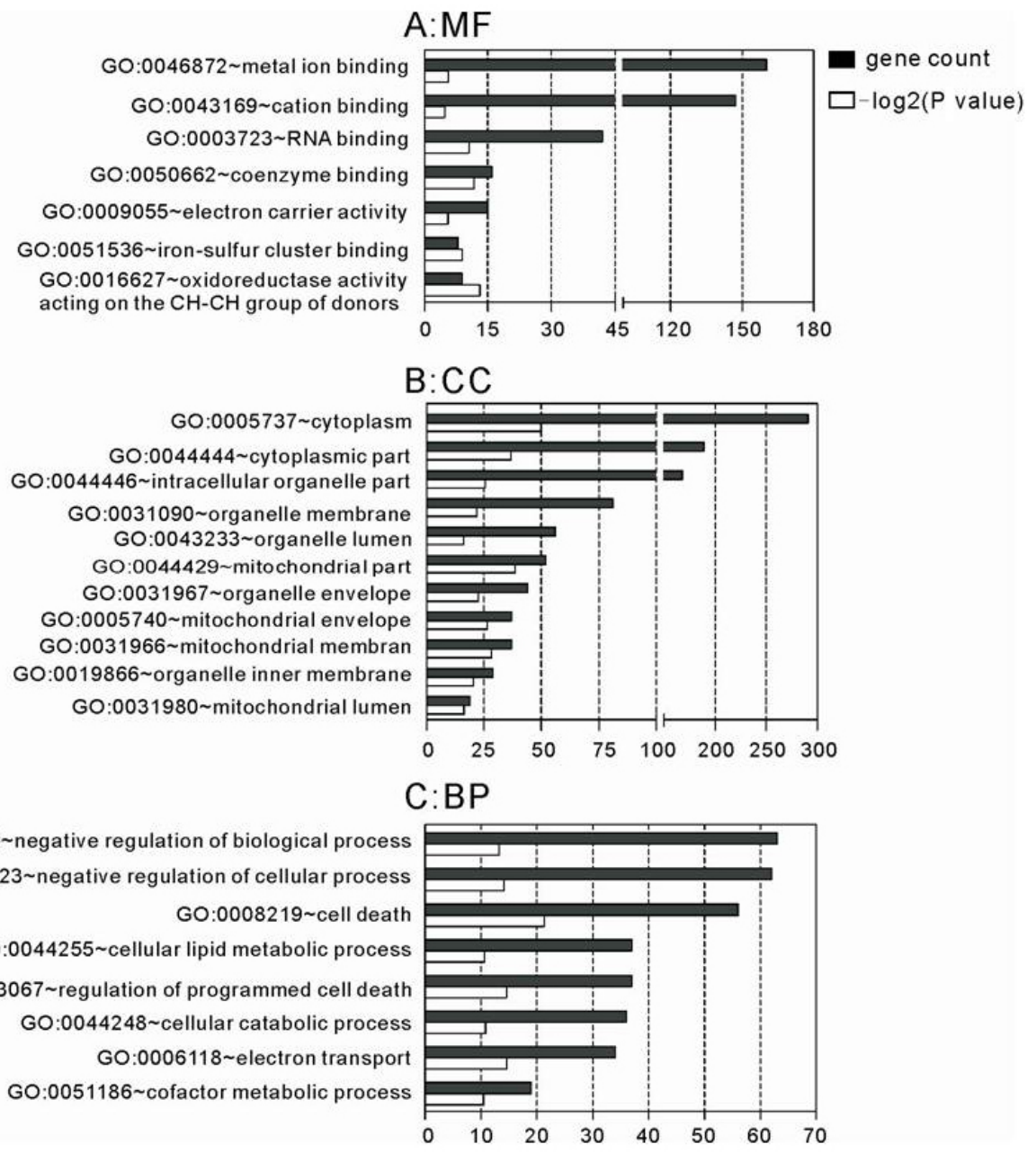

Figure 2. GO categories for differential co-expression probes in diabetic insulin-sensitive tissues. Filled and open bars represent gene number and negative logarithm of $\mathrm{P}$ value based on 2, respectively. A longer bar represents a smaller P value. A. Molecular Function (MF). B. Cell Component of GO (CC). C. Biological Process of GO (BP). Only the classes that were over-represented by the EASE score $(\mathrm{P}<0.05)$ and set at intermediate coverage and specificity, i.e., the GO categories with DAVID P $<0.05$, are shown. 


\section{KEEG pathway analysis for genes differentially expressed in various insulin-sensitive tissues from a human with T2D}

To identify the most commonly dysregulated metabolism pathways in diabetic insulinsensitive tissues, we performed KEEG pathway analysis using two tools with different platforms, DAVID and GSEA. The DAVID databases found that, with the exception of the mROT signaling pathway, the PPAR $\gamma$ and insulin signaling pathways were associated with $\mathrm{T} 2 \mathrm{D}$, and these differentially expressed genes of insulin-sensitive tissues were also enriched for energyrelated KEGG pathways, including fatty acid metabolism $(\mathrm{P}=0.0016)$, oxidative phosphorylation $(\mathrm{P}=0.0114)$, TCA cycle $(\mathrm{P}=0.028)$, glycolysis/gluconeogenesis $(\mathrm{P}=0.0209)$; results of which are shown in Figure 3A. Similar results were also obtained with GSEA (Figure 3B).

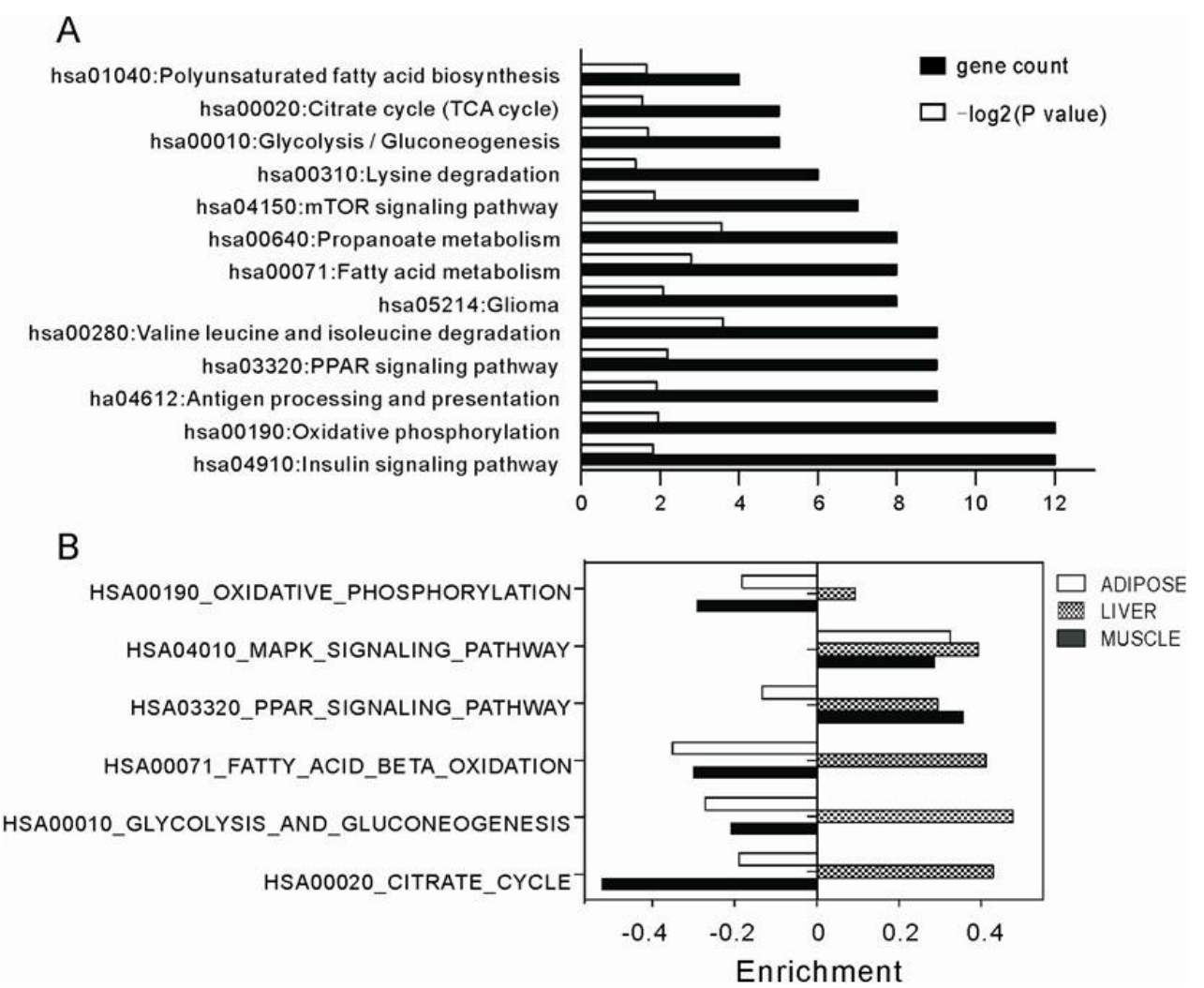

Figure 3. KEGG pathway-enrichment analysis for differential expression genes in insulin-sensitive tissues of human with T2D. A. The enrichment of KEGG pathway offered by DAVID with minimum of 2 LocusLinks. B. The common enriched KEGG pathways of insulin-sensitive tissues by GSEA, and positive and negative number of enrichment score represented upregulation and downregulation, respectively.

\section{Expression of energy-related metabolism genes within insulin-sensitive tissues of a human with T2D}

The microarray data of energy-related metabolism genes that were differentially ex- 
pressed in all insulin-sensitive tissues examined were analyzed further (Table 1). Genes related to glycolysis/gluconeogenesis, the TCA cycle, and electron transport were mostly downregulated in diabetic skeletal muscle and visceral adipose tissue, while they were upregulated in diabetic liver. Genes related to fatty acid beta oxidation were downregulated in diabetic liver and adipose tissue, while they were upregulated and downregulated in diabetic muscle. These results were in line with our GSEA findings. Furthermore, many genes overlapped in relation to their involvement in pathways. The TCA cycle genes $S D H A, S D H B$, and $N N T$ and fatty acid beta oxidation genes $A C A D M$ and $G C D H$ were also involved in the electron transport chain, and glycolysis/gluconeogenesis genes $A L D H 2$ and $P D H B$ were involved in fatty acid beta oxidation and TCA cycle.

Table 1. Genes differentially co-regulated in diabetic insulin-sensitive tissues.

\begin{tabular}{|c|c|c|c|c|}
\hline \multirow[t]{2}{*}{ Gene symbol } & \multirow[t]{2}{*}{ Gene name } & \multicolumn{3}{|c|}{ Fold-change } \\
\hline & & Liver & Fatty & Muscle \\
\hline \multicolumn{5}{|c|}{ Glycolysis/gluconeogenesis } \\
\hline ALDH2* & Aldehyde dehydrogenase 2 family & 2.83 & -1.94 & -1.60 \\
\hline PGM1 & Phosphoglucomutase 1 & 3.15 & -1.83 & -2.18 \\
\hline ENO1 & Enolase 1 & 7.23 & -1.53 & -2.19 \\
\hline PDHB & Pyruvate dehydrogenase beta & 3.62 & -1.63 & -2.81 \\
\hline GPI & Glucose phosphate isomerase & -3.10 & -1.51 & -1.69 \\
\hline \multicolumn{5}{|c|}{ Fatty acid beta oxidation } \\
\hline ACADM* & Acyl-co-enzyme A dehydrogenase, C-4 to C-12 straight chain & 8.20 & -2.63 & -2.00 \\
\hline HADHSC* & L-3-hydroxyacyl-co-enzyme A dehydrogenase, short chain & 5.59 & -2.13 & -2.70 \\
\hline ACAT1 & Acetyl-co-enzyme A acetyltransferase 1 & 2.19 & -1.69 & -1.62 \\
\hline ACSL3 & Achaete-scute complex homolog 3 & 1.59 & -1.90 & -2.33 \\
\hline ACSL1* & Achaete-scute complex homolog 1 & 4.54 & -1.94 & -2.23 \\
\hline $\mathrm{GCDH}^{*}$ & Glutaryl-co-enzyme A dehydrogenase & 4.25 & -1.90 & -2.30 \\
\hline ACAA1 & Acetyl-co-enzyme A acyltransferase 1 & 3.28 & -1.75 & -1.90 \\
\hline \multicolumn{5}{|c|}{ Tricarboxylic acid cycle } \\
\hline $\mathrm{ACO} 2$ & Aconitase 2 , mitochondrial & 1.72 & -2.20 & -1.95 \\
\hline SDHA* & Succinate dehydrogenase, complex, subunit A & 1.94 & -2.29 & -1.51 \\
\hline SDHB* & Succinate dehydrogenase, complex, subunit B & 2.33 & -2.16 & -2.21 \\
\hline $\mathrm{NNT}^{*}$ & Nicotinamide nucleotide transhydrogenase & 2.46 & -2.11 & -3.20 \\
\hline SUCLG2 & Succinate-CoA ligase, GDP-forming, beta subunit & 3.03 & -1.52 & -2.01 \\
\hline \multicolumn{5}{|c|}{ Electron transport chain ${ }^{\#}$} \\
\hline NDUFB2* & NADH dehydrogenase (ubiquinone) 1 beta subcomplex, 2 & 2.40 & -1.29 & -1.46 \\
\hline NDUFB5* & NADH dehydrogenase (ubiquinone) 1 beta subcomplex, 5 & 2.62 & -1.54 & -1.76 \\
\hline NDUFC2* & NADH dehydrogenase (ubiquinone) 1 , subcomplex , 2 & 3.12 & -1.53 & -1.84 \\
\hline COX8A* & Cytochrome c oxidase subunit $8 \mathrm{~A}$ & 2.30 & -1.66 & -2.17 \\
\hline COX15* & Cytochrome c oxidase assembly protein (yeast) & 1.34 & -1.43 & -1.39 \\
\hline CYB5A & Cytochrome B5 type A & 1.82 & -2.24 & -1.84 \\
\hline CYP2C9 & Cytochrome P450, family 2, subfamily C, polypeptide 9 & 5.60 & -2.19 & -2.52 \\
\hline CYP2E1 & Cytochrome P450, family 2, subfamily E, polypeptide 1 & 1.62 & -1.73 & -3.14 \\
\hline MT1X & Metallothionein 1X & 1.65 & -4.54 & -6.23 \\
\hline PCYOX1 & Phenyl cysteine oxidase 1 & 7.09 & -2.09 & -3.48 \\
\hline UQCRC2* & Biquinol-cytochrome c reductase core protein II & 2.11 & -2.06 & -1.93 \\
\hline UQCRFS1* & Ubiquinol-cytochrome c reductase, Rieske iron-sulfur polypeptide 1 & 2.03 & -1.71 & -2.43 \\
\hline ATP5J* & ATP synthase, $\mathrm{H}+$ transporting, mitochondrial F0 complex, subunit F6 & 2.70 & -1.56 & -1.64 \\
\hline ATP5G3* & ATP synthase, $\mathrm{H}+$ transporting, mitochondrial F0 complex, subunit $\mathrm{C} 3$ (subunit 9) & 1.92 & -1.71 & -2.18 \\
\hline ATP5A1* & ATP synthase, $\mathrm{H}+$ transporting, mitochondrial F1 complex, alpha subunit 1 & 1.91 & -2.31 & -1.94 \\
\hline
\end{tabular}

*Expression of genes identified by quantitative real-time PCR. ${ }^{*}$ A total of 34 electron transport chain genes were dysregulated in insulin-sensitive tissues of type 2 diabetic by microarray; a portion of these genes were listed.

\section{Confirmation of microarray expression data in selected energy-related metabolism genes by qRT-PCR}

To validate microarray gene expression data, we used qRT-PCR to detect the expression of genes that were selected on the basis of biological interest or their fold-change between 
an independent cohort of T2D patients and controls. The expression of 18 genes is shown in Figure 4. For these genes, apart from $A L D H 2$ and $C O X 15$, the qRT-PCR expression data were consistent with the microarray expression findings. Gene expression data from the microarrays, therefore, likely reflect actual expression levels to some extent.

A

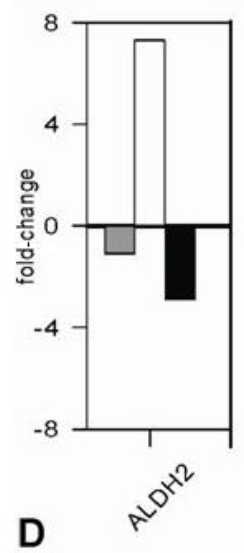

B

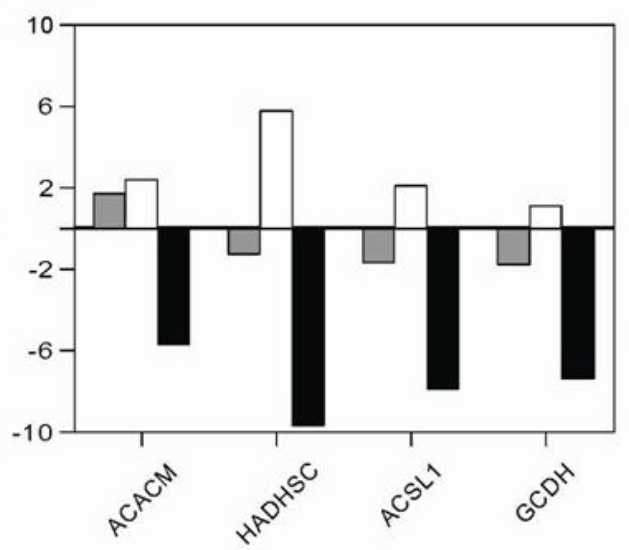

C

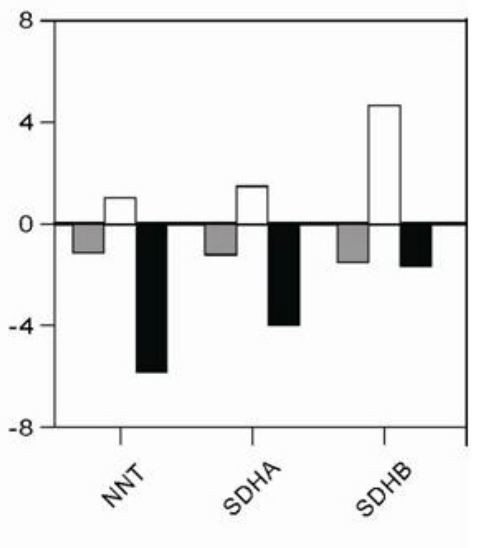

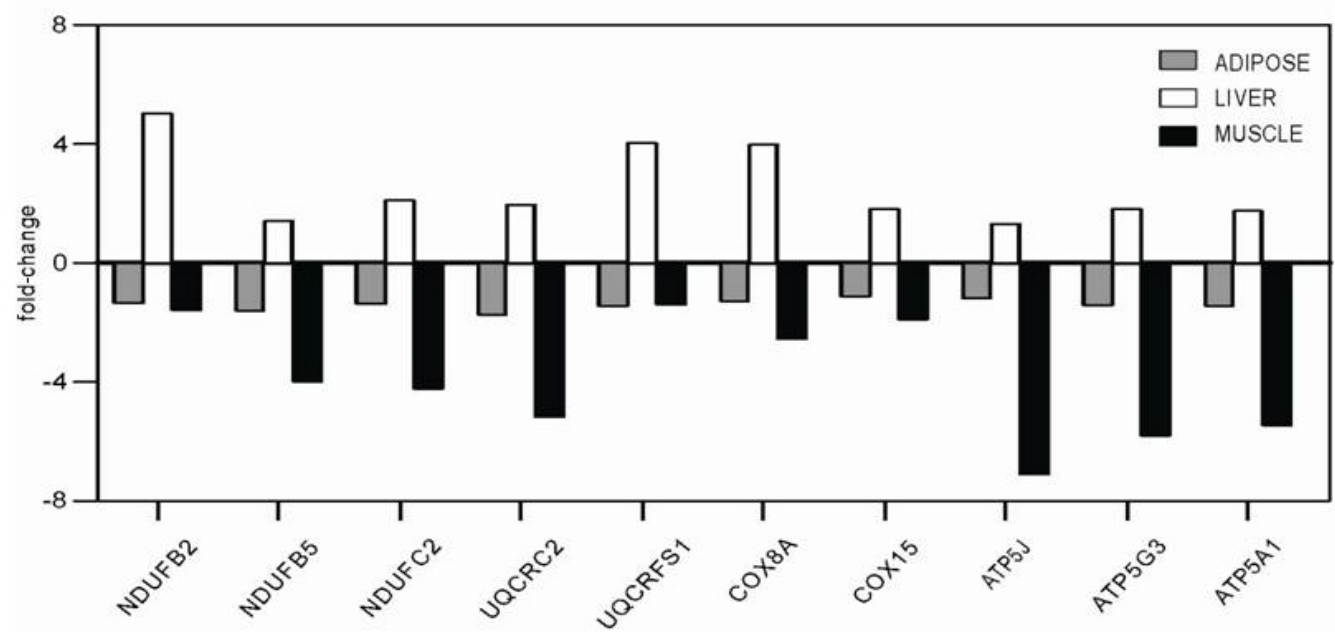

Figure 4. Ratio of mRNA expression of energy-related metabolic genes in diabetic adipose tissue, liver, skeletal muscle $(N=4)$ versus corresponding control tissues $(N=5)$. A. Gene for glycolysis/gluconeogenesis; $\mathbf{B}$. genes for fatty acid beta oxidation; C. genes for tricarboxylic acid cycle; D. genes for electron transport chain. Changes in expression of genes in diabetic insulin-sensitive tissues are expressed relative to controls. SYBR Green assays were used for mRNA quantification with $\beta$-actin as internal control.

\section{DISCUSSION}

In the present study, we used a combination approach of microarray and bioinformatic technologies to investigate the differential expression of genes in diabetic adipose tissue, skeletal muscle, and liver. We recognized that microarray approaches were limited by multiple 
comparison caveats and false positives. Furthermore, many observed changes in gene expression were of relatively low magnitude, and the small subject number in our study limits our power to detect differences between groups. Despite these limitations, two different bioinformatic tools focused on alterations in gene expression at the level of biological pathways or coregulated gene sets, rather than individual genes. Regardless, observed microarray expression differences were validated for representative genes by qRT-PCR (Figure 4).

To the best of our knowledge, gene expression profiles comparing diabetic insulinsensitive tissues have not yet been identified using high-density oligonucleotide arrays, because of the difficulty in obtaining samples from a clinic. In this study, we comprehensively evaluated the gene expression profiles of three insulin-sensitive tissues of patients with and without T2D (Figure 1). Our findings revealed that the vast majority of genes were downregulated in diabetic adipose and liver tissues, while within muscle tissue, the number of upregulated genes was greater than the number of downregulated.

Genes identified to be differentially expressed between diabetic insulin-sensitive tissues and control tissues may reflect either the pathophysiology of insulin resistance or other metabolic factors. We performed GO and KEEG pathway analysis on the simultaneously dysregulated genes in diabetic insulin-sensitive tissues. GO molecular functions such as co-enzyme binding, electron carrier activity, and oxidoreductase activities were found to be enriched (Figure 2A). Moreover, genes expressed differentially in all the insulin-sensitive tissues of a T2D patient that we examined were related to mitochondria in the cellular component of GO terms, including the mitochondrial part, mitochondrial lumen, mitochondrial membrane, and mitochondrial envelope (Figure 2B). Furthermore, GO biological process terms, including electron transport, the cellular lipid metabolic process, and the cellular catabolic process, were significantly overrepresented (Figure 2C). These data indicate that certain GO categories for the probes differentially expressed in all tissues were significantly over-represented, namely those related to energy metabolism, which, therefore, may contribute to the development of T2D.

In addition, KEGG pathway analysis indicated that the dysregulated pathways common to all the tissues examined were electron transport, TCA cycle, glycolysis and gluconeogenesis, and fatty acid beta oxidation, suggesting that the dysregulation of energy metabolism in multiple insulin-sensitive tissues may contribute to the development of T2D. Furthermore, by GSEA, it became evident that the TCA cycle, oxidative phosphorylation, and the glycolysis/ gluconeogenesis pathway were downregulated in diabetic skeletal muscle and visceral adipose tissue, whereas they were upregulated in diabetic liver, and fatty acid beta oxidation was downregulated in all three insulin-sensitive tissues, consistent with previous reports (Mootha et al., 2003; Patti et al., 2003; Dahlman et al., 2006; Delarue and Magnan, 2007; Misu et al., 2007; Schrauwen and Hesselink, 2008). Taken together, our data indicate that genes that are differentially expressed in all of these sensitive-insulin tissues share some common characteristics of metabolism, mainly suggesting that energy metabolism could be involved in insulin resistance.

Dysregulation of free fatty acid (FFA) metabolism is a key event responsible for the development of insulin resistance and T2D (Delarue and Magnan, 2007). In this study, FFA beta oxidation was downregulated in muscle and adipose tissue of T2D, and, thus, could contribute to triglyceride accumulation. In diabetic liver, however, FFA beta oxidation was upregulated, and subsequently generated acetyl-CoA and activated pyruvate carboxylase, phosphoenol pyruvate carboxykinase, $N A D H$, and ATP that is used in gluconeogenesis. In healthy subjects, lipolysis is tightly regulated in such a way that the partition of energy fuels (glucose and FFAs) is generated based on the energy needs of different tissues (Langin, 2006). Con- 
versely, in diseases such as obesity and T2D, FFA flux is chronically increased, and it is this permanent increase in FFA availability that is thought to induce and/or aggravate liver and skeletal muscle insulin resistance (DeFronzo, 2004; Blaak, 2005; Kovacs and Stumvoll, 2005).

We also showed that genes involved in the TCA cycle, including ACO2, SDHA, $S D H B, P D H B$, and $S U C L G 2$, were downregulated in both diabetic adipose tissue and skeletal muscle. These observed decreases in expression would be expected to inhibit the conversion of pyruvate to acetyl $\mathrm{CoA}$ and then to direct pyruvate to either oxaloacetate by pyruvate carboxylase or to lactate. These findings were consistent with previously published data that suggested reduced TCA cycle flux in type 2 diabetic patients (Befroy et al., 2007; Schrauwen and Hesselink, 2008). The upregulation of the TCA cycle in diabetic liver, however, may be due to the excess presence of the metabolites glucose and FFA. In insulin resistance and T2D, hepatic gluconeogenesis was also found to be elevated, which would likewise increase the requirement for more ATP for catalytic actions, and, thus, naturally the expression of the TCA cycle was increased. Furthermore, our results indicated that genes involved in the electron transport chain were downregulated in diabetic skeletal muscle and visceral adipose tissue, while they were upregulated in diabetic liver, findings that are consistent with previous reports (Mootha et al., 2003; Patti et al., 2003; Dahlman et al., 2006; Misu et al., 2007; Takamura et al., 2008), and confirm that mitochondrial OXPHOS may play an important role in the pathophysiology of T2D (Petersen et al., 2003; Lowell and Shulman, 2005).

It is known that most of the energy from the oxidation of carbohydrates and fatty acids comes from the generation of reducing equivalents from the Krebs cycle, and the subsequent generation of ATP via redox reactions of the electron transport chain. The pattern of dysregulation for the Krebs cycle, fatty acid $\beta$-oxidation, and electron transport varies among diabetic insulin-sensitive tissues, because they play different roles in glucose and lipid metabolism. Dysregulated energy metabolism, however, could be viewed as the causative feature behind dysregulated adenosine triphosphate production that would affect in insulin signaling leading to defects in glucose transport, inflammation, and oxidative stress, ultimately resulting in insulin resistance. Determining whether the dysregulation of energy metabolism is the cause or a consequence of $\mathrm{T} 2 \mathrm{D}$ requires further study.

In conclusion, our data suggest that several genes involved in energy metabolism pathways are differentially expressed in diabetic adipose tissue, skeletal muscle, and liver. Dysregulation of adenosine triphosphate production may affect insulin activity in peripheral tissues. Although the expression differences observed for individual genes did not meet statistical significance, pattern analysis demonstrated that genes encoding proteins mainly related to mitochondrial oxidative metabolism were differentially expressed between insulin-sensitive $\mathrm{T} 2 \mathrm{D}$ and control tissues. These findings, therefore, may provide insight into the pathogenesis of insulin resistance in T2D and help to identify candidate genes and pathways that could serve as new targets for diagnostic and therapeutic interventions. Further experimental studies, however, are required before drawing any definitive conclusions on this topic.

\section{ACKNOWLEDGMENTS}

We thank Feizhen $\mathrm{Wu}, \mathrm{PhD}$, from the Laboratory of Epigenetics, Institutes of Biomedical Sciences, Fudan University for excellent bioinformatic analysis. Research supported by the National Natural Science Foundation of China (\#81100589 and \#30670999) and the Scientific Research Project of Shanghai First People's Hospital (\#10B19). 


\section{REFERENCES}

Befroy DE, Petersen KF, Dufour S, Mason GF, et al. (2007). Impaired mitochondrial substrate oxidation in muscle of insulin-resistant offspring of type 2 diabetic patients. Diabetes 56: 1376-1381.

Blaak EE (2005). Metabolic fluxes in skeletal muscle in relation to obesity and insulin resistance. Best Pract. Res. Clin. Endocrinol. Metab. 19: 391-403.

Castro-Chavez F, Yechoor VK, Saha PK, Martinez-Botas J, et al. (2003). Coordinated upregulation of oxidative pathways and downregulation of lipid biosynthesis underlie obesity resistance in perilipin knockout mice: a microarray gene expression profile. Diabetes 52: 2666-2674.

Colombo M, Gregersen S, Kruhoeffer M, Agger A, et al. (2005). Prevention of hyperglycemia in Zucker diabetic fatty rats by exercise training: effects on gene expression in insulin-sensitive tissues determined by high-density oligonucleotide microarray analysis. Metabolism 54: 1571-1581.

Colombo M, Kruhoeffer M, Gregersen S, Agger A, et al. (2006). Energy restriction prevents the development of type 2 diabetes in Zucker diabetic fatty rats: coordinated patterns of gene expression for energy metabolism in insulinsensitive tissues and pancreatic islets determined by oligonucleotide microarray analysis. Metabolism 55: 43-52.

Dahlman I, Forsgren M, Sjogren A, Nordstrom EA, et al. (2006). Downregulation of electron transport chain genes in visceral adipose tissue in type 2 diabetes independent of obesity and possibly involving tumor necrosis factor-alpha. Diabetes 55: 1792-1799.

DeFronzo RA (2004). Dysfunctional fat cells, lipotoxicity and type 2 diabetes. Int. J. Clin. Pract. (Suppl): 9-21.

Delarue J and Magnan C (2007). Free fatty acids and insulin resistance. Curr. Opin. Clin. Nutr. Metab. Care 10: 142-148.

Dennis G Jr, Sherman BT, Hosack DA, Yang J, et al. (2003). DAVID: Database for Annotation, Visualization, and Integrated Discovery. Genome Biol. 4: 3.

Huang dW, Sherman BT and Lempicki RA (2009). Systematic and integrative analysis of large gene lists using DAVID bioinformatics resources. Nat. Protoc. 4: 44-57.

Kasuga M (2006). Insulin resistance and pancreatic beta cell failure. J. Clin. Invest. 116: 1756-1760.

Keller MP, Choi Y, Wang P, Davis DB, et al. (2008). A gene expression network model of type 2 diabetes links cell cycle regulation in islets with diabetes susceptibility. Genome Res. 18: 706-716.

Kovacs P and Stumvoll M (2005). Fatty acids and insulin resistance in muscle and liver. Best Pract. Res. Clin. Endocrinol. Metab. 19: 625-635.

Langin D (2006). Control of fatty acid and glycerol release in adipose tissue lipolysis. C. R. Biol. 329: 598-607.

Lee RK, Hittel DS, Nyamandi VZ, Kang L, et al. (2012). Unconventional microarray design reveals the response to obesity is largely tissue specific: analysis of common and divergent responses to diet-induced obesity in insulinsensitive tissues. Appl. Physiol. Nutr. Metab. 37: 257-268.

Lowell BB and Shulman GI (2005). Mitochondrial dysfunction and type 2 diabetes. Science 307: 384-387.

Misu H, Takamura T, Matsuzawa N, Shimizu A, et al. (2007). Genes involved in oxidative phosphorylation are coordinately upregulated with fasting hyperglycaemia in livers of patients with type 2 diabetes. Diabetologia 50: 268-277.

Mootha VK, Lindgren CM, Eriksson KF, Subramanian A, et al. (2003). PGC-1alpha-responsive genes involved in oxidative phosphorylation are coordinately downregulated in human diabetes. Nat. Genet. 34: 267-273.

Patti ME, Butte AJ, Crunkhorn S, Cusi K, et al. (2003). Coordinated reduction of genes of oxidative metabolism in humans with insulin resistance and diabetes: Potential role of PGC1 and NRF1. Proc. Natl. Acad. Sci. U. S. A. 100: 8466-8471.

Petersen KF, Befroy D, Dufour S, Dziura J, et al. (2003). Mitochondrial dysfunction in the elderly: possible role in insulin resistance. Science 300: 1140-1142.

Schrauwen P and Hesselink MK (2008). Reduced tricarboxylic acid cycle flux in type 2 diabetes mellitus? Diabetologia 51: 1694-1697.

Subramanian A, Tamayo P, Mootha VK, Mukherjee S, et al. (2005). Gene set enrichment analysis: a knowledge-based approach for interpreting genome-wide expression profiles. Proc. Natl. Acad. Sci. U. S. A. 102: 15545-15550.

Suh YH, Kim Y, Bang JH, Choi KS, et al. (2005). Analysis of gene expression profiles in insulin-sensitive tissues from pre-diabetic and diabetic Zucker diabetic fatty rats. J. Mol. Endocrinol. 34: 299-315.

Takamura T, Misu H, Matsuzawa-Nagata N, Sakurai M, et al. (2008). Obesity upregulates genes involved in oxidative phosphorylation in livers of diabetic patients. Obesity 16: 2601-2609.

Way JM, Harrington WW, Brown KK, Gottschalk WK, et al. (2001). Comprehensive messenger ribonucleic acid profiling reveals that peroxisome proliferator-activated receptor gamma activation has coordinate effects on gene expression in multiple insulin-sensitive tissues. Endocrinology 142: 1269-1277.

Wisloff U, Najjar SM, Ellingsen O, Haram PM, et al. (2005). Cardiovascular risk factors emerge after artificial selection for low aerobic capacity. Science 307: 418-420. 